\title{
ON THE EXISTENCE OF CENTRAL SEQUENCES IN SUBFACTORS
}

\author{
DIETMAR H. BISCH
}

\begin{abstract}
We prove a relative version of [Co1, Theorem 2.1] for a pair of type $\mathrm{II}_{1}$-factors $N \subset M$. This gives a list of necessary and sufficient conditions for the existence of nontrivial central sequences of $M$ contained in the subfactor $N$. As an immediate application we obtain a result by Bédos [Be, Theorem A], showing that if $N$ has property $\Gamma$ and $G$ is an amenable group acting freely on $N$ via some action $\sigma$, then the crossed product $N \times{ }_{\sigma} G$ has property $\Gamma$. We also include a proof of a relative Mc Duff-type theorem (see [McD, Theorems 1,2 and 3]), which gives necessary and sufficient conditions implying that the pair $N \subset M$ is stable.
\end{abstract}

\section{INTRODUCTION}

The property $\Gamma$ for a factor of type II $_{1}$ was introduced by Murray-von Neumann (see $[\mathrm{MvN}])$ to distinguish two different classes of factors. It describes an asymptotic commutativity property of the algebra. A stronger property was later considered by Mc Duff (see [McD]) in order to construct more examples of factors. Both concepts turned out to be essential. Connes used them in his fundamental papers (see [Co1, Co2]), not only to prove the uniqueness of the hyperfinite $\mathrm{II}_{1}$-factor $R$, but also to classify the automorphisms of $R$. He gives some surprising alternative characterizations of these properties in [Col, Theorem 2.1].

In this paper we study necessary and sufficient conditions for the existence of nontrivial central sequences in a type $\mathrm{II}_{1}$-factor $M$ that are contained in a subfactor $N \subset M$. Our work is motivated by Problem 3 in [Jo] and the related generating problem for pairs of hyperfinite factors with finite index (see [Po3, Oc]): Jones asks in [Jo] for conditions implying that the pair $N \subset M$ is stable, i.e. isomorphic to the pair $N \bar{\otimes} R \subset M \bar{\otimes} R$. If $N \subset M$ are hyperfinite and have the generating property, then the pair $N \subset M$ is stable. A necessary condition for stability and hence for the generating property as well, is the existence of nontrivial central sequences of $M$ contained in $N$.

In the first section we prove a relative version of Connes' Theorem 2.1 in [Co1]. We show that the existence of central sequences for the ambient factor $M$ that are actually contained in $N$ is equivalent to the existence of a singular state $\varphi$ of $M$ that is invariant under a finitely generated subgroup of Int $M$

Received by the editors June 7, 1989.

1980 Mathematics Subject Classification (1985 Revision). Primary 46L35; Secondary 46L10. 
and factors through the conditional expectation $E_{N}$ from $M$ onto $N$. This is equivalent to saying that there is no nonzero compact operator of $B\left(L^{2}(M, \tau)\right)$ contained in the $C^{*}$-algebra generated by $M, M^{\prime}$ and $e_{N}$, where $e_{N}$ denotes the orthogonal projection from $L^{2}(M, \tau)$ onto $L^{2}(N, \tau)$. The proof of our theorem closely follows the ideas of Connes' proof.

In the second section we give some applications to crossed products. We show that if $N$ is a separable $\mathrm{II}_{1}$-factor with property $\Gamma$ and $G$ is an amenable group acting freely on $N$, then the crossed product $N \times{ }_{\sigma} G$ also has property $\Gamma$. Popa proves this result in [Po2] for $G=\mathbf{Z}$ and conjectures it for a general amenable group, which was shown to be true by Bédos [Be, Theorem A]. Bédos uses a technique involving the decomposition of the crossed product [Be, Proposition $3]$. We derive the result as an immediate application of the main theorem in $\S 1$.

In the third section we prove a relative version of Mc Duff's theorem (see [McD, Theorems 1, 2 and 3]. This result, showing that $N \subset M$ is stable if and only if $N$ contains noncommuting central sequences of $M$, was probably noticed by specialists, but no detailed proof seems to exist in the literature.

Notation. $M$ denotes a separable $\mathrm{II}_{1}$-factor acting on the Hilbert space $L^{2}(M, \tau)$, where $\tau$ is the normal faithful normalized trace on $M . N \subset$ $M$ is a subfactor and $E_{N}: M \mapsto N$ the unique conditional expectation with $\tau \circ E_{N}=\tau . \quad e_{N}$ denotes the orthogonal projection from $L^{2}(M, \tau)$ onto $L^{2}(N, \tau) \subset L^{2}(M, \tau)$, and $\|x\|_{2}=\tau\left(x^{*} x\right)^{1 / 2}, x \in M$, is the Hilbert norm as usual. Furthermore $J$ will be the canonical involution in $L^{2}(M, \tau)$, i.e. $J x=x^{*}$ for all $x \in M$. We denote by $\otimes$ the algebraic tensor product and by $\bar{\otimes}$ the von Neumann algebra tensor product. $B\left(L^{2}(M, \tau)\right)$ is the algebra of bounded linear operators on $L^{2}(M, \tau)$.

Acknowledgment. I am very grateful to Professor Sorin Popa, who suggested this problem to me.

\section{Central sequences in subfactors}

We recall that $M$ has property $\Gamma$ if for given elements $x_{1}, \ldots, x_{n} \in M$ and $\varepsilon>0$ there is a unitary $u \in M$ with $\tau(u)=0$ such that $\left\|\left[x_{i}, u\right]\right\|_{2} \leq \varepsilon$, $1 \leq i \leq n$. If $N \subset M$ is a subfactor, we are interested in conditions that asssure that the unitary $u$ is actually contained in $N$. We obtain the following theorem:

Theorem 1.1. Let $M$ be a separable $\mathrm{II}_{1}$-factor with subfactor $N \subset M$. Then the following conditions are equivalent:

$1^{\circ}$. For any elements $x_{1}, \ldots, x_{n} \in M$ and $\varepsilon>0$ there is a unitary $u \in N$ with $\tau(u)=0$ and $\left\|\left[x_{i}, u\right]\right\|_{2} \leq \varepsilon$ for all $i=1, \ldots, n$.

$2^{\circ}$. For any finitely generated group $G \subset$ Int $M$ there is a nonnormal $G$ invariant state $\varphi \in M^{*}$ with $\varphi \circ E_{N}=\varphi$. 
$2^{\prime \circ}$. Let $N \subset M_{0} \subset M$ be a $\|\cdot\|_{2}$-dense $*$-subalgebra of $M$.

For any elements $x_{1}, \ldots, x_{n} \in M_{0}$ there is a nonnormal state $\varphi \in M^{*}$ with $\varphi\left(x x_{i}\right)=\varphi\left(x_{i} x\right)$ for all $x \in M, 1 \leq i \leq n$, and $\varphi \circ E_{N}=\varphi$.

$3^{\circ}$. For any operators $x_{1}, \ldots, x_{n} \in M$ there is a sequence $\left(\xi_{k}\right)_{k \in \mathbf{N}} \subset$ $L^{2}(N, \tau),\left\|\xi_{k}\right\|_{2}=1,\left|\left\langle\xi_{k}, 1\right\rangle\right| \nrightarrow 1$ as $k \rightarrow \infty$, such that $\left\|\left[x_{i}, \xi_{k}\right]\right\|_{2}$ $\rightarrow 0$.

$4^{\circ}$. The $C^{*}$-algebra $C^{*}\left(M, M^{\prime}, e_{N}\right)$ generated by $M, M^{\prime}, e_{N}$ in $B\left(L^{2}(M, \tau)\right)$ does not contain any nonzero compact operator, i.e.

$$
C^{*}\left(M, M^{\prime}, e_{N}\right) \cap \mathscr{K}\left(L^{2}(M, \tau)\right)=\{0\} .
$$

The proof of this theorem will use the following lemmata.

Lemma 1.2. Let $N \subset M$ be $\mathrm{II}_{1}$-factors as in Theorem 1.1, satisfying $2^{\circ}$. Then for any elements $x_{1}, \ldots, x_{n} \in M$ and $\varepsilon>0$ there is a nonzero projection $e \in N$ with $\tau(e) \leq \varepsilon$ and

$$
\left\|\left[x_{i}, e\right]\right\|_{2} \leq \varepsilon\|e\|_{2}, \quad 1 \leq i \leq n .
$$

Proof. We may assume that the given elements $x_{i}$ are in fact unitaries, denoted by $u_{i}, 1 \leq i \leq n$. By hypothesis there is a singular state $\varphi \in M^{*}$ with

$$
\begin{aligned}
& \varphi=\varphi \circ \text { Ad } u_{i}, \quad 1 \leq i \leq n, \\
& \varphi=\varphi \circ E_{N} .
\end{aligned}
$$

Then there is a projection $f \in N$ with $\varphi(f)=1, \tau(f)<\varepsilon, 0<\varepsilon<\frac{1}{2}$ (see [Ta1]). Set

$$
\begin{aligned}
V & :=\{\psi \text { state on } M \mid \psi(f) \geq 1-\varepsilon\} \subset M^{*}, \\
W & :=\left\{\left(\psi-\psi \circ E_{N}, \psi-\psi \circ \operatorname{Ad} u_{1}, \ldots, \psi-\psi \circ \operatorname{Ad} u_{n}\right) \mid \psi \in V \cap M_{*}\right\} .
\end{aligned}
$$

Identifying as usual $\left(M^{n}\right)^{*} \cong\left(M^{*}\right)^{n},\left(\left(M_{*}\right)^{n}\right)^{*} \cong M^{n}$, we conclude that zero belongs to the $\sigma\left(\left(M^{*}\right)^{n+1}, M^{n+1}\right)$-closure of $W$, because $\varphi$ is in the $\sigma\left(M^{*}, M\right)$-closure of $V \cap M_{*}$. Zero is of course in $\left(M_{*}\right)^{n+1}$, and since $W$ is a (nonempty) convex subset of $\left(M_{*}\right)^{n+1}$, a separation argument shows that zero is in fact in the norm-closure of $W$ in $\left(M_{*}\right)^{n+1}$. Thus there is a $\psi \in V \cap M_{*}$ such that

$$
\left\|\psi-\psi \circ E_{N}\right\|, \quad\left\|\psi-\psi \circ \operatorname{Ad} u_{i}\right\| \leq \varepsilon, \quad 1 \leq i \leq n .
$$

The fact $\psi(f) \geq 1-\varepsilon$ implies that $\psi$ is basically supported on $f M f$, i.e. if we set

$$
\tilde{\psi}(x):=\frac{\psi(f x f)}{\psi(f)}, \quad x \in M,
$$

we get a normal state $\tilde{\psi}$ with $\|\psi-\tilde{\psi}\| \leq 3 \varepsilon^{1 / 2}$ (as in [Col, Lemma 2.4]). Define

$$
\psi^{\prime}(x):=\tilde{\psi}\left(E_{N}(x)\right)
$$


then $\psi^{\prime}$ is a positive normal state with

$$
\left\|\psi-\psi^{\prime}\right\| \leq\left\|(\tilde{\psi}-\psi) \circ E_{N}\right\|+\left\|\psi-\psi \circ E_{N}\right\| \leq 3 \varepsilon^{1 / 2}+\varepsilon .
$$

Note that $\psi^{\prime} \circ E_{N}=\psi^{\prime}$, supp $\psi^{\prime} \leq f$. Since $\psi^{\prime}$ is positive and normal, there is a unique $\xi \in L^{1}(M, \tau), \xi \geq 0$ with $\psi^{\prime}(x)=\tau(x \xi), x \in M . E_{N}$ extends to a continuous $N-N$-bimodule map from $L^{1}(M, \tau)$ to $L^{1}(N, \tau)$, hence

$$
\begin{aligned}
\tau(x \xi) & =\psi^{\prime}(x)=\psi^{\prime}\left(E_{N}(x)\right)=\tau\left(E_{N}(x) \xi\right) \\
& =\tau\left(E_{N}(x) E_{N}(\xi)\right)=\tau\left(x E_{N}(\xi)\right), \quad \forall x \in M .
\end{aligned}
$$

But this shows that $\xi \in L^{1}(N, \tau)$. Thus $h:=\xi^{1 / 2}$ is in $L^{2}(N, \tau)^{+},\|h\|_{2}=1$, and

$$
\psi^{\prime}(x)=\tau(x \xi)=\omega_{h}(x)=\langle x h, h\rangle .
$$

We have $\operatorname{supp} h \leq f$ and by the Powers-Størmer inequality

$$
\begin{aligned}
\left\|u_{i} h u_{i}^{*}-h\right\|_{2}^{2} & \leq \| \psi^{\prime}-\psi^{\prime} \circ \text { Ad } u_{i} \| \\
& \leq\left\|\psi^{\prime}-\psi\right\|+\| \psi-\psi \circ \text { Ad } u_{i}\|+\|\left(\psi-\psi^{\prime}\right) \circ \text { Ad } u_{i} \| \\
& \leq 9 \varepsilon^{1 / 2}, \quad 1 \leq i \leq n .
\end{aligned}
$$

Using Connes' trick [Co1, Theorem 1.2.2], for $\delta=6 n\left(3 \varepsilon^{1 / 4}\right)^{1 / 8}<1$ (i.e. $\varepsilon$ small enough), we can find a $t \in \mathbf{R}, t>0$, such that the spectral projection $E_{t}(h) \in N\left(E_{t}(h)\right.$ denotes as usual the spectral projection $\left.\chi_{(t, \infty)}(h)\right)$ is nonzero and satisfies

$$
\left\|E_{t}\left(u_{i} h u_{i}^{*}\right)-E_{t}(h)\right\|_{2} \leq \delta\left\|E_{t}(h)\right\|_{2}, \quad 1 \leq i \leq n .
$$

Putting $e=E_{t}(h)$, we get

$$
\left\|\left[u_{i}, e\right]\right\|_{2} \leq \delta\|e\|_{2},
$$

since $E_{t}\left(u_{i} h u_{i}^{*}\right)=u_{i} e u_{i}^{*}, 1 \leq i \leq n$. We have clearly

$$
\tau(e) \leq \tau(\operatorname{supp} h) \leq \tau(f)<\varepsilon,
$$

and $e \in N$ since $h \in L^{2}(N, \tau)$. Q.E.D.

We use the following relative version of [Co1, Lemma 2.6]-the proof is the same.

Lemma 1.3. Let $M$ be a $\mathrm{II}_{1}$-factor, $N \subset M$ a subfactor, $\omega$ a free ultrafilter on $\mathbf{N}, N^{\omega}$ and $M^{\omega}$ the usual ultrapower algebras and $u_{1}, \ldots, u_{n}$ unitary operators in $M$. Then if the commutant of $u_{1}, \ldots, u_{n}$ in $N^{\omega}$ is finite dimensional, we can find unitary operators $u_{n+1}, \ldots, u_{q}$ in $M$ such that the commutant of $u_{1}, \ldots, u_{q}$ in $N^{\omega}$ is trivial.

Similar to [Co1, Lemma 2.5], we need the next lemma for the proof of the implication $2^{\circ}\left(\right.$ resp. $\left.2^{\prime \circ}\right) \Rightarrow 1^{\circ}$. 
Lemma 1.4. Let $N \subset M_{0} \subset M$ satisfy statement $2^{\prime \circ}$ in Theorem 1.1. Then the reduced algebras $N_{p} \subset p M_{0} p \subset M_{p}$ also satisfy $2^{\prime \circ}$, where $p$ denotes a nontrivial projection in $N$.

Proof. We use Connes' argument: since $N$ is a $\mathrm{II}_{1}$-factor there is a projection $f \in N$ with $f \leq p, \tau(f)=\frac{1}{k}, k \in \mathbf{N}$. It is now easy to construct a type $\mathrm{I}_{k^{-}}$ subfactor $P$ of $N$ with minimal projection $f$. $P$ is generated by two unitaries $v_{1}, v_{2} \in N$.

Let $x_{1}, \ldots, x_{n} \in p M_{0} p \subset M_{0}$. Then by hypothesis there is a singular state $\varphi \in M^{*}$ with $\varphi\left(x_{i} x\right)=\varphi\left(x x_{i}\right), 1 \leq i \leq n, \varphi\left(v_{i} x\right)=\varphi\left(x v_{i}\right), i=1,2, \forall x \in$ $M$, and $\varphi=\varphi \circ E_{N}$. The restriction of $\varphi$ to $M_{p}$ is the desired singular functional. Q.E.D.

Lemma 1.4 shows in particular that if $N \subset M$ satisfy $2^{\circ}$, then the reduced algebras $N_{p} \subset M_{p}$ also satisfy $2^{\circ}$.

Proof of Theorem 1.1 (see [Co1, Proof of Theorem 2.1]).

We prove the following chain of implications: $1^{\circ} \Rightarrow 4^{\circ} \Rightarrow 3^{\circ} \Rightarrow 2^{\circ} \Rightarrow 1^{\circ}$,

$2^{\circ} \Rightarrow 2^{\circ} \Rightarrow 1^{\circ} \Rightarrow 2^{\circ}$.

$1^{\circ} \Rightarrow 4^{\circ}$ : The proof of this implication is based on Connes' idea.

$1 .{ }^{\circ}$ implies the existence of a nontrivial central sequence of unitaries $\left(u_{i}\right)_{i \in \mathbf{N}} \subset$ $N$ with $\tau\left(u_{i}\right)=0, i \in \mathbf{N},\left\|\left[x, u_{i}\right]\right\|_{2} \rightarrow 0$ as $i \rightarrow \infty$ for all $x \in M$. Since $M$ is a factor, $C^{*}\left(M, M^{\prime}, e_{N}\right)$ is irreducible and therefore we have either

$$
\mathscr{K}(H) \cap C^{*}\left(M, M^{\prime}, e_{N}\right)=\{0\} \quad \text { or } \mathscr{K}(H) \subset C^{*}\left(M, M^{\prime}, e_{N}\right) .
$$

If $4^{\circ}$ does not hold, the one-dimensional projection $\pi: H \mapsto \operatorname{span} \hat{1}, \xi \mapsto$ $\langle\xi, \hat{1}\rangle \hat{1}$, where $\hat{1}$ is as usual the cyclic and separating vector in $L^{2}(M, \tau)$, is contained in $C^{*}\left(M, M^{\prime}, e_{N}\right)$. Hence there are words $a_{1}^{(1)} \ldots a_{n_{1}}^{(1)}, \ldots, a_{1}^{(k)} \ldots$ $a_{n_{k}}^{(k)} \in C^{*}\left(M, M^{\prime}, e_{N}\right)$ with $a_{i}^{(j)} \in M \cup M^{\prime} \cup\left\{e_{N}\right\}$ such that

$$
\left\|\pi-\sum_{i=1}^{k} a_{1}^{(i)} \cdots a_{n_{i}}^{(i)}\right\|<\frac{\varepsilon}{2} .
$$

The elements in $M$ commute with those in $M^{\prime}$, but not necessarily with $e_{N}$. We suppose that every word is written in a form where all the elements in $M$ that occur in the word are moved as far to the left as possible. Note that $u_{i}$ commutes with $M^{\prime}$ and $e_{N}$. We can assume that every word contains an element in $M$ (otherwise the process described below is not necessary). Let $a_{s_{i}}^{(i)}$ denote the first element of $M$ that occurs in the word $a_{1}^{(i)} \cdots a_{n_{i}}^{(i)}$ (from left to right); then

$$
\left\|\sum_{i=1}^{k} a_{1}^{(i)} \cdots\left[a_{s_{i}}^{(i)}, u_{j}\right] a_{s_{i}+1}^{(i)} \cdots a_{n_{i}}^{(i)} \hat{1}\right\|_{2} \rightarrow 0 \quad \text { as } j \rightarrow \infty,
$$


i.e.

$$
\left\|\sum_{i=1}^{k} a_{1}^{(i)} \cdots a_{s_{i}}^{(i)} u_{j} a_{s_{i}+1}^{(i)} \cdots a_{n_{i}}^{(i)} \hat{1}-u_{j} \sum_{i=1}^{k} a_{1}^{(i)} \cdots a_{n_{i}}^{(i)} \hat{1}\right\|_{2} \rightarrow 0 \quad \text { as } j \rightarrow \infty,
$$

which implies that

$$
\left\|\sum_{i=1}^{k} a_{1}^{(i)} \cdots a_{s_{i}}^{(i)} u_{j} a_{s_{i}+1}^{(i)} \cdots a_{n_{i}}^{(i)} \hat{\mathrm{1}}\right\|_{2} \rightarrow\left\|\sum_{i=1}^{k} a_{1}^{(i)} \cdots a_{n_{i}}^{(i)} \hat{\mathrm{1}}\right\|_{2} \quad \text { as } j \rightarrow \infty .
$$

(1) shows that

$$
1-\frac{\varepsilon}{2} \leq\left\|\sum_{i=1}^{k} a_{1}^{(i)} \cdots a_{n_{i}}^{(i)} \hat{1}\right\|_{2} \leq 1+\frac{\varepsilon}{2} .
$$

This together with (2) implies that we can fix a $j_{0} \geq 1$ such that

$$
\left\|\sum_{i=1}^{k} a_{1}^{(i)} \cdots a_{s_{i}}^{(i)} u_{j} a_{s_{i}+1}^{(i)} \cdots a_{n_{i}}^{(i)} \hat{1}\right\|_{2} \geq 1-\varepsilon, \quad \forall j \geq j_{0} .
$$

The idea is now to move $u_{j}$ through the word to the right. $u_{j}$ commutes with all $a_{s_{i}}^{(i)}, a_{s_{i}+1}^{(i)}, \ldots$ until it hits the next element $a_{r_{i}}^{(i)}$ in $M$. Then we repeat the process described above, and we can find an index $j_{1}$ such that for all $j \geq j_{1} \geq j_{0}$, we have

$$
\left\|\sum_{i=1}^{k} a_{1}^{(i)} \cdots a_{r_{i}}^{(i)} u_{j} a_{r_{i}+1}^{(i)} \cdots a_{n_{i}}^{(i)} \hat{1}\right\|_{2} \geq 1-\varepsilon-\frac{\varepsilon}{2} .
$$

This procedure stops after at most $n=\max \left\{n_{1}, \ldots, n_{k}\right\}$ steps, so we get an index $j_{n} \geq 1$ such that for all $j \geq j_{n}$, we have

$$
\left\|\sum_{i=1}^{k} a_{1}^{(i)} \cdots a_{n_{i}}^{(i)} u_{j} \hat{1}\right\|_{2} \geq 1-\varepsilon \sum_{n=0}^{\infty}\left(\frac{1}{2}\right)^{n}>\varepsilon,
$$

if $0<\varepsilon<\frac{1}{3}$. On the other hand we get from (1)

$$
\frac{\varepsilon}{2}>\left\|\pi\left(\hat{u}_{j}\right)-\sum_{i=1}^{k} a_{1}^{(i)} \cdots a_{n_{i}}^{(i)} u_{j} \hat{1}\right\|_{2}=\left\|\sum_{i=1}^{k} a_{1}^{(i)} \cdots a_{n_{i}}^{(i)} u_{j} \hat{1}\right\|_{2},
$$

which contradicts $(3)$ for all $j \geq j_{n}$. This shows that $C^{*}\left(M, M^{\prime}, e_{N}\right)$ cannot contain any nonzero compact operator.

$4^{\circ} \Rightarrow 3^{\circ}$ : Suppose $3^{\circ}$ does not hold. This implies that there are unitaries $u_{1}, \ldots, u_{n}$ in $M$ such that there is no sequence $\left(\xi_{k}\right)_{k \in \mathbf{N}} \subset L^{2}(N, \tau),\left\langle\xi_{k}, \hat{1}\right\rangle$ $=0,\left\|\xi_{k}\right\|_{2}=1$ with $\left\|\left(u_{i}-J u_{i}^{*} J\right) \xi_{k}\right\|_{2} \rightarrow 0,1 \leq i \leq n$.

We show that $C^{*}\left(M, M^{\prime}, e_{N}\right)$ must contain the orthogonal projection from $L^{2}(M, \tau)$ onto $\mathbf{C} \hat{1}$. Consider

$$
T:=\sum_{i=1}^{n} e_{N} u_{i} J u_{i} J e_{N}
$$


Then $T \in C^{*}\left(M, M^{\prime}, e_{N}\right)$ and adjoining $u_{i}^{*}, 1 \leq i \leq n$, to the $u_{1}, \ldots, u_{n}$ if necessary, we may assume $T$ is selfadjoint. Then $\|T\|=n$ and $T \hat{1}=$ $n \hat{1} . T-n \cdot 1$ is invertible as an operator from the complement of $\hat{1}$ in $L^{2}(N, \tau)$ into itself. Indeed, because if we assume $T-n \cdot 1$ is not invertible, then there is a sequence $\left(\xi_{k}\right)_{k \in \mathbf{N}} \subset L^{2}(N, \tau),\left\langle\xi_{k}, \hat{1}\right\rangle=0,\left\|\xi_{k}\right\|_{2}=1$ with

$$
\left\|(T-n 1) \xi_{k}\right\|_{2} \rightarrow 0 \quad \text { as } k \rightarrow \infty .
$$

This would imply $\left\|T \xi_{k}\right\|_{2} \rightarrow n$. But

$$
\left\|T \xi_{k}\right\|_{2} \leq\left\|\sum_{i=1}^{n} u_{i} J u_{i} J \xi_{k}\right\|_{2} \leq n,
$$

and hence $\left\|\sum_{i=1}^{n} u_{i} J u_{i} J \xi_{k}\right\|_{2} \rightarrow n$. The strict convexity of $L^{2}(M, \tau)$ implies then that $\left\|\left(u_{i}-J u_{i}^{*} J\right) \xi_{k}\right\|_{2} \rightarrow 0,1 \leq i \leq n$, which contradicts the choice of the $u_{1}, \ldots, u_{n}$. Therefore $T-n \cdot 1$ is invertible on the orthogonal complement of $\mathbf{C} \hat{1}$ in $L^{2}(N, \tau)$, hence $n$ is an isolated simple point of the spectrum of $T$, so the corresponding spectral projection is one-dimensional and in $C^{*}\left(M, M^{\prime}, e_{N}\right)$, which contradicts $4^{\circ}$.

$\underline{3^{\circ} \Rightarrow 2^{\circ}}$ : Given $u_{1}, \ldots, u_{n}$ unitaries in $M$, we want to show that there is a nonnormal state $\varphi \in M^{*}$ with $\varphi \circ$ Ad $u_{i}=\varphi, 1 \leq i \leq n, \varphi \circ E_{N}=\varphi$. The proof is the same as Connes', but for the convenience of the reader we briefly sketch the argument. Fix a free ultrafilter $\omega$ of $\mathbf{N}$. By Lemma 1.3 we only have to consider the following two cases:

(a) $\left\{u_{1}, \ldots, u_{n}\right\}^{\prime} \cap N^{\omega}=\mathbf{C}$. Let $\left(\xi_{k}\right)_{k \in \mathbf{N}} \subset L^{2}(N, \tau)$ be as in $3^{\circ}$, i.e. $\left\|\xi_{k}\right\|_{2}=1,\left\langle\xi_{k}, \hat{1}\right\rangle=0, k \in \mathbf{N},\left\|\left[u_{i}, \xi_{k}\right]\right\|_{2} \rightarrow 0$ as $k \rightarrow \infty, 1 \leq i \leq n$. As in [Col], using the hypothesis on the relative commutant, there is an $\varepsilon>0$ and a subsequence $\left(\eta_{k}\right)_{k \in \mathbf{N}}$ of the sequence $\left(\xi_{k}\right)_{k \in \mathbf{N}}$ such that $\tau\left(E_{k}\left(\left|\eta_{k}\right|^{2}\right)\left|\eta_{k}\right|^{2}\right) \geq \varepsilon$ for all $k \in \mathbf{N}$. Put $\varphi_{k}=\tau\left(\cdot\left|\eta_{k}\right|^{2}\right)$ and $e_{k}=E_{k}\left(\left|\eta_{k}\right|^{2}\right)$. The $\varphi_{k}$ are states on $M$ with $\varphi_{k} \circ E_{N}=\varphi_{k}$ (since $\eta_{k} \in L^{2}(N, \tau)$ ) and $\left\|\left[\varphi_{k}, u_{i}\right]\right\| \rightarrow 0$ as $k \rightarrow \infty, 1 \leq i \leq n$. Akemann's theorem [Ak, Theorem 2.3] shows that $\left(\varphi_{k}\right)_{k \in \mathbf{N}}$ is not weakly relatively compact in $M_{*}$ since $\varphi_{k}\left(e_{k}\right) \geq \varepsilon$ for all $k \in \mathbf{N}$, but $e_{k} \rightarrow 0$ strongly. Any nonnormal $\varphi$ in the weak closure of $\left\{\varphi_{k}\right\}_{k \in \mathbf{N}}$ in $M^{*}$ will do.

(b) $\left\{u_{1}, \ldots, u_{n}\right\}^{\prime} \cap N^{\omega}$ is infinite-dimensional. Thus this commutant contains an infinite-dimensional abelian von Neumann subalgebra and hence contains nonzero projections $e_{k}$ with $\tau_{\omega}\left(e_{k}\right) \leq \frac{1}{k}, k \in \mathbf{N}$. Each $e_{k}$ is represented by a sequence of projections $\left(f_{k}\right)_{k \in \mathbf{N}} \subset N$ with $f_{k} \neq 0, \tau\left(f_{k}\right) \leq$ $\frac{1}{k},\left\|\left[u_{i}, f_{k}\right]\right\|_{2} \leq \frac{1}{k}\left\|f_{k}\right\|_{2} \forall i, k$. Let $\varphi_{k}=\tau\left(\cdot \frac{f_{k}}{\tau\left(f_{k}\right)}\right)$; then as in (a) we have $\varphi_{k}=\varphi_{k} \circ E_{N}$, and we can find a weak limit of the $\varphi_{k}$ with the desired properties.

$\underline{2}^{\circ} \Rightarrow 1^{\circ}$ : We prove this implication using a maximality argument, slightly different from the one in [Co1], similar to the ones in [CoFW and Po2]. Let 
$u_{1}, \ldots, u_{n}$ be unitaries in $M$ and $\varepsilon>0$. Consider

$R:=\{f \mid f$ projection in $N$ such that:

$$
\begin{aligned}
& \text { (1) } \tau(f) \leq \frac{1}{2} \text { and } \\
& \text { (2) } \left.\left\|(1-f) u_{i} f-f u_{i}(1-f)\right\|_{2} \leq \varepsilon\|f\|_{2}, 1 \leq i \leq n\right\} \text {. }
\end{aligned}
$$

$R$ is clearly inductively ordered and nonempty, so we can take a maximal element $f \in R$. We show that $\tau(f)=\frac{1}{2}$. If not, there is a $\delta>0$ with $\tau(f)+\delta \leq$ $\frac{1}{2}(\delta<\varepsilon)$. Lemmas 1.4 and 1.2 applied to $N_{1-f} \subset M_{1-f},(1-f) u_{i}(1-f)$, $1 \leq i \leq n$, and $\delta>0$, give a nonzero projection $e \in N_{1-f}$ with $\tau(e) \leq \delta$ and

$$
\left\|\left[(1-f) u_{i}(1-f), e\right]\right\|_{2} \leq \delta\|e\|_{2}, \quad 1 \leq i \leq n .
$$

Put $f_{0}:=f+e$, which is a projection in $N$, strictly larger than $f$, and satisfies $\tau\left(f_{0}\right) \leq \tau(f)+\delta \leq \frac{1}{2}$. We compute

$$
\begin{aligned}
\left\|\left[u_{i}, f_{0}\right]\right\|_{2}^{2} & =\left\|(1-f-e) u_{i} f-f u_{i}(1-f-e)+(1-f) u_{i} e-e u_{i}(1-f)\right\|_{2}^{2}, \\
& =\left\|(1-f-e) u_{i} f-f u_{i}(1-f-e)\right\|_{2}^{2}+\left\|\left[(1-f) u_{i}(1-f), e\right]\right\|_{2}^{2}, \\
& \leq\left\|(1-f) u_{i} f-f u_{i}(1-f)\right\|_{2}^{2}+\left\|\left[(1-f) u_{i}(1-f), e\right]\right\|_{2}^{2}, \\
& =\varepsilon^{2}\|f\|_{2}^{2}+\delta^{2}\|e\|_{2}^{2} \leq \varepsilon^{2}\|f+e\|_{2}^{2}=\varepsilon^{2}\left\|f_{0}\right\|_{2}^{2} .
\end{aligned}
$$

Therefore

$$
\left\|\left(1-f_{0}\right) u_{i} f_{0}-f_{0} u_{i}\left(1-f_{0}\right)\right\|_{2} \leq \varepsilon\left\|f_{0}\right\|_{2}, \quad 1 \leq i \leq n,
$$

which contradicts the maximality of $f$.

Put $u:=2(1-f)-1$, then $\tau(u)=0, u$ is a unitary in $N$ and $\left\|\left[u_{i}, u\right]\right\|_{2} \leq$ $2 \cdot \frac{\varepsilon}{2}=\varepsilon, 1 \leq i \leq n$.

$2^{\circ} \Leftrightarrow 2^{\prime \circ}: 2^{\circ} \Rightarrow 2^{\prime \circ}$ is trivial. Conversely, we show $2^{\prime \circ} \Rightarrow 1^{\circ}$, which is equivalent to $2^{\circ}$. Using Lemma 1.4 and Lemma 1.2, the same maximality argument used above works, because the projections in the set $R$ are in $N$ and $N$ is contained in $M_{0}$. We get therefore statement $1^{\circ}$ with $M_{0}$ in place of $M$. This is clearly enough to show $1^{\circ}$ since $u$ is bounded. Q.E.D.

\section{APPLICATIONS TO CROSSED PRODUCTS}

Throughout this section $N$ denotes a separable $\mathrm{II}_{1}$-factor and $G$ a countable (discrete) amenable group acting freely on $N$ via $\sigma: G \mapsto$ Aut $N$. Applying Theorem 1.1 to the pair of factors $N \subset N \times{ }_{\sigma} G$, we get a simple proof of the following result due to Bédos [Be, Theorem $\mathrm{A}]$ :

Theorem 2.1. Let $N$ be a separable $\mathrm{II}_{1}$-factor with property $\Gamma$ and $G$ an amenable group acting freely on $N$ via some action $\sigma$. Then given any $x_{1}, \ldots, x_{n} \in$ $N \times_{\sigma} G$ and $\varepsilon>0$, there is a unitary $u \in N$ with $\tau(u)=0$ and $\left\|\left[u, x_{i}\right]\right\|_{2}<\varepsilon$ for all $i=1, \ldots, n$. In particular, there are nontrivial central sequences of $N \times_{\sigma} G$ contained in $N$ and $N \times_{\sigma} G$ has property $\Gamma$.

Proof. Let $M:=N \times_{\sigma} G$ and denote by $u_{g}$ the unitaries implementing the action of $G$ on $N$, i.e. $u_{g} x u_{g}^{*}=\sigma_{g}(x), x \in N$. Set $M_{0}:=\left\{\sum_{\text {finite }} x_{g} u_{g}, x_{g} \in\right.$ $N\}$; then with $N \subset M_{0} \subset M$ we are in the situation of statement $2^{\prime \circ}$ of 
Theorem 1.1. Given unitaries $u_{1}, \ldots, u_{n} \in N$ and $u_{g_{1}}, \ldots, u_{g_{k}}$, we have to construct a nonnormal state $\varphi \in M^{*}$ with $\varphi\left(x u_{i}\right)=\varphi\left(u_{i} x\right), \varphi\left(x u_{g_{j}}\right)=$ $\varphi\left(u_{g_{j}} x\right), \forall x \in M, \forall i, j$ and $\varphi \circ E_{N}=\varphi$.

Let $\left(F_{n}\right)_{n \in \mathbf{N}}$ be an increasing sequence of finite subset of $G$ with $\bigcup_{n=1}^{\infty} F_{n}=$ $G$. Since $G$ is amenable, given $F_{n}$ and $\varepsilon_{n}>0$, there is a finite set $K_{n} \subset G$ with $\left|K_{n} F_{n} \backslash K_{n}\right|<\varepsilon_{n}\left|K_{n}\right|$. We choose $\varepsilon_{n} \searrow 0$. Given $u_{1}, \ldots, u_{n}, \sigma_{g}\left(u_{i}\right)$, $g \in K_{j}, 1 \leq i \leq n$, unitaries in $N$, there is a singular state $\varphi_{j} \in N^{*}$ which is invariant under these unitaries [Co1, Theorem 2.1]. We put

$$
\varphi_{K_{j}}(x)=\frac{1}{\left|K_{j}\right|} \sum_{g \in K_{j}} \varphi_{j} \circ E_{N}\left(u_{g} x u_{g}^{*}\right), \quad x \in M .
$$

By construction we have $\varphi_{K_{j}}\left(x u_{i}\right)=\varphi_{K_{j}}\left(u_{i} x\right), \forall x \in M, 1 \leq i \leq n$, and the $\varphi_{K_{j}}$ are clearly singular states on $M$ which factor through $E_{N}$. The choice of the sets $\left(K_{n}\right)_{n \in \mathbf{N}}$ implies that

$$
\left\|\left[\varphi_{K_{j}}, u_{g}\right]\right\| \rightarrow 0 \quad \text { as } j \rightarrow \infty, \quad \forall g \in G .
$$

Let $\varphi$ be a $\sigma\left(M^{*}, M\right)$-limit point of $\left\{\varphi_{K_{j}}\right\}_{j \in \mathbf{N}}$; then by [Ta2, Chapter III, Proposition 5.8] $\varphi$ is still singular and has, by the construction of the $\varphi_{K_{j}}$, the desired invariance properties. Applying $2^{\prime \circ}$ of Theorem 1.1 gives the result. Q.E.D.

We also mention here a remark, essentially showing that the set of subfactors of a given $\mathrm{II}_{1}$-factor $M$, that contain a fixed subfactor $N$ and have nontrivial central sequences in $N$, is inductively ordered.

Proposition 2.2. Let $N, M_{n}, M$ be $\mathrm{II}_{1}$-factors with $M$ separable such that $N \subset M_{n} \subset M$ for all $n \in \mathbf{N}$ and $M_{n} \nearrow M$ (i.e. ${\overline{\bigcup_{n=1}^{\infty} M_{n}}}^{w}=M$ ). If there are nontrivial central sequences for $M_{n}$ contained in $N$ for all $n \in \mathbf{N}$, then there are nontrivial central sequences for $M$ contained in $N$.

Proof. The hypothesis imply that $1^{\circ}$ of Theorem 1.1 holds for all pairs $N \subset$ $M_{n}, n \in \mathbf{N}$. We will check condition $1^{\circ}$ for $N \subset M$. Given $x_{1}, \ldots, x_{n} \in M$ and $\varepsilon>0$, there is an $n_{0} \geq 1$ and $x_{1}^{0}, \ldots, x_{n}^{0} \in M_{n_{0}}$ such that $\left\|x_{i}-x_{i}^{0}\right\|_{2}<\frac{\varepsilon}{2}$, $1 \leq i \leq n$. But for $x_{1}^{0}, \ldots, x_{n}^{0}$ there is a unitary $u \in N$ with $\tau(u)=0$ and $\left\|\left[x_{i}^{0}, u\right]\right\|_{2}<\frac{\varepsilon}{2}$, thus $\left\|\left[x_{i}, u\right]\right\|_{2}<\varepsilon, \quad 1 \leq i \leq n$. Q.E.D.

\section{A relative Mc Duff-type theorem}

Mc Duff considers in [McD] separable $\mathrm{II}_{1}$-factors $M$ which are isomorphic to $M \bar{\otimes} R, R$ the hyperfinite $\mathrm{II}_{1}$-factor. This property is stronger than property $\Gamma$ and is in fact equivalent to the noncommutativity of the algebra $M^{\prime} \cap M^{\omega}, \omega$ a free ultrafilter in $\mathbf{N}$. Using Mc Duff's methods we prove a relative version of her theorem for a pair of (separable) $\mathrm{II}_{1}$-factors $N \subset M$. The theorem gives a necessary and sufficient condition implying that the pair $N \subset M$ is isomorphic to the pair $N \bar{\otimes} R \subset M \bar{\otimes} R$. 
Theorem 3.1. Let $M$ be a separable $\mathrm{II}_{1}$-factor with subfactor $N \subset M$. Then the following conditions are equivalent:

$1^{\circ}$. Given $x_{1}, \ldots, x_{n} \in M$ and $\varepsilon>0$, there is a $\mathrm{I}_{2}$-subfactor $M_{2 \times 2}$ in $N$ with matrix units $\left\{e_{i j}\right\}_{1 \leq i, j \leq 2}$ such that

$$
\left\|\left[e_{i j}, x_{k}\right]\right\|_{2}<\varepsilon, \quad 1 \leq k \leq n, \quad 1 \leq i, j \leq 2 .
$$

$2^{\circ} . N \subset M \cong N \bar{\otimes} R \subset M \bar{\otimes} R$, i.e. there is an isomorphism $\Phi: M \mapsto M \bar{\otimes} R$ (onto) with $\Phi(N)=N \bar{\otimes}$.

$3^{\circ} . M^{\prime} \cap N^{\omega}$ is noncommutative, where $\omega$ is a free ultrafilter in $\mathbf{N}$.

Since the proof follows closely Mc Duff's ideas, we avoid details where our proof coincides with Mc Duff's.

Proof. We show $1^{\circ} \Leftrightarrow 2^{\circ}$ and $1^{\circ} \Leftrightarrow 3^{\circ}$.

$1^{\circ} \Rightarrow 2^{\circ}$ : In Lemmas 3.2 and 3.3 below we prove two results similar to [McD, Theorems 1, 2]. The proofs are essentially the ones given by Mc Duff adapted to the situation $N \subset M$.

Lemma 3.2. Let $\left(M_{k}\right)_{k=1}^{\infty}$ be a sequence of $\mathrm{I}_{2}$-subfactors of $N$ with matrix units $\left\{e_{i j}^{k}\right\}$ such that

(i) $M_{j}$ commutes with $M_{k}, j \neq k$.

(ii) $\left(e_{i j}^{k}\right)_{k=1}^{\infty}$ is a central sequence in $M$ for all $(i, j)$ fixed.

Then there is a hyperfinite $\mathrm{II}_{1}$-factor $R \subset N$ with

$$
N \cong R \bar{\otimes}\left(R^{\prime} \cap N\right), \quad M \cong R \bar{\otimes}\left(R^{\prime} \cap M\right)
$$

and thus $N \subset M \cong N \bar{\otimes} R \subset M \bar{\otimes} R$.

Proof. We use the following well-known fact: If $K \subset M$ is a $\mathrm{I}_{k}$-subfactor, then

$$
\left\|x-E_{K^{\prime} \cap M}(x)\right\|_{2} \leq k^{\frac{3}{2}} \sup _{1 \leq i, j \leq k}\left\|\left[e_{i j}, x\right]\right\|_{2},
$$

where $\left\{e_{i j}\right\}_{1 \leq i, j \leq k}$ are matrix units in $K$ and $E_{K^{\prime} \cap M}$ is the conditional expectation $M \mapsto K^{\prime} \cap M$.

Let $\left(x_{n}\right)_{n=1}^{\infty}\left(\right.$ resp. $\left.\left(y_{n}\right)_{n=1}^{\infty}\right)$ be a $\|\cdot\|_{2}$-dense sequence in $M$ (resp. $\left.N\right)$. Using (4) and (ii) of the hypothesis, we can find for given $x_{1}, \ldots, x_{k}, y_{1}, \ldots, y_{k}$ and $\varepsilon=2^{-k}$ a matrix-algebra $M_{n_{k}} \in\left\{M_{k}, k=1,2, \ldots\right\}$ such that

$$
\left\|x_{i}-E_{M_{n_{k}}^{\prime} \cap M}\left(x_{i}\right)\right\|_{2},\left\|y_{i}-E_{M_{n_{k}}^{\prime} \cap N}\left(y_{i}\right)\right\|_{2}<2^{-k}, \quad 1 \leq i \leq k
$$

Notice that $M_{n_{k}} \subset N$. This provides a subsequence $\left\{M_{n_{k}}\right\}_{k=1}^{\infty}$ of $\left\{M_{k}\right\}_{k=1}^{\infty}$ and we set

$$
R:=\bar{\bigotimes}_{k=1}^{\infty} M_{n_{k}}
$$

$R$ splits $M$ and $N$ simultaneously. For this it is enough to show that $x_{i} \in$ $\left(R \cup\left(R^{\prime} \cap M\right)\right)^{\prime \prime}$ (resp. $\left.y_{i} \in\left(R \cup\left(R^{\prime} \cap N\right)\right)^{\prime \prime}\right)$ for all $i \in \mathbf{N}$. Similar to [McD, 
inequality (7)], we get

(6) $\left\|x-E_{\left(\otimes_{i=n+1}^{\infty} M_{n_{i}}\right)^{\prime} \cap M}(x)\right\|_{2} \leq \sum_{i=n+1}^{\infty}\left\|x-E_{M_{n_{i}}^{\prime} \cap M}(x)\right\|_{2}, \quad x \in M, n \in \mathbf{N}$.

So if we fix $x_{i_{0}}$ and $y_{i_{0}}$, we get from (6) using (5) for $n>i_{0}, j_{0}$ :

$$
\left\|x_{i_{0}}-E_{\left(\otimes_{i=n+1}^{\infty} M_{n_{i}}\right)^{\prime} \cap M}\left(x_{i_{0}}\right)\right\|_{2} \leq \sum_{i=n+1}^{\infty} 2^{-i} \rightarrow 0 \quad \text { as } n \rightarrow \infty,
$$

and similarly for $y_{i_{0}}$. But $E_{\left(\otimes_{i=n+1}^{\infty} M_{n_{i}}\right)^{\prime} \cap M}\left(x_{i_{0}}\right)$ (resp. $E_{\left(\otimes_{i=n+1}^{\infty} M_{n_{i}}\right)^{\prime} \cap N}\left(y_{i_{0}}\right)$ ) is contained in $\left(\bigotimes_{i=1}^{n} M_{n_{i}} \cup\left(R^{\prime} \cap M\right)\right)^{\prime \prime}$ (resp. $\left.\left(\bigotimes_{i=1}^{n} M_{n_{i}} \cup\left(R^{\prime} \cap N\right)\right)^{\prime \prime}\right)$, which shows that $N \cong R \bar{\otimes}\left(R^{\prime} \cap N\right), M \cong R \bar{\otimes}\left(R^{\prime} \cap M\right)$. Analyzing these standard isomorphisms, we get the desired result, since $R \cong R \bar{\otimes}$. This ends the proof of Lemma 3.2.

As in $[\mathrm{McD}$, Theorem 2], we need a second lemma, which will prove the implication $1^{\circ} \Rightarrow 2^{\circ}$ together with Lemma 3.2.

Lemma 3.3. Let $\left(M_{k}\right)_{k=1}^{\infty}$ be a sequence of $\mathrm{I}_{2}$-subfactors of $N$ with matrix units $\left\{e_{i j}^{k}\right\}$ forming central sequences in $M$. Then there is a sequence $\left(N_{k}\right)_{k=1}^{\infty}$ of mutually commuting $\mathrm{I}_{2}$-subfactors in $N$ with matrix units $\left\{g_{i j}^{k}\right\}$, which also form central sequences in $M$.

Proof. Mc Duff's proof of Theorem 2 in [McD] applies completely; her construction never leaves $N$.

This ends the proof of $1^{\circ} \Rightarrow 2^{\circ}$.

$\underline{2^{\circ} \Rightarrow 1^{\circ}}$ : The desired $\mathrm{I}_{2}$-subfactor can be constructed in $R$ and then transported to $N$ via the given isomorphism.

$1^{\circ} \Rightarrow 3^{\circ}$ : Using the separability of $N$ we obtain a sequence of $\mathrm{I}_{2}$-subfactors of $N$ with matrix units $\left\{e_{i j}^{k}\right\}$ such that

$$
\left\|\left[x, e_{i j}^{k}\right]\right\|_{2} \rightarrow 0 \quad \text { as } k \rightarrow \infty, \quad \forall x \in M,(i, j) \text { fixed } .
$$

But then $\left(e_{12}^{n}\right)\left(e_{21}^{n}\right) \neq\left(e_{21}^{n}\right)\left(e_{12}^{n}\right)$ and the elements $\left(e_{12}^{n}\right)$ and $\left(e_{21}^{n}\right)$ are nontrivial in $N^{\omega}$.

$3^{\circ} \Rightarrow 1^{\circ}$ : The argument is the same as Mc Duff's. For the sake of completeness we recall briefly the steps.

Let $I_{0}=\left\{\left(x_{n}\right) \in l^{\infty}(\mathbf{N}, M) \mid \lim _{n \rightarrow \infty}\left\|x_{n}\right\|_{2}=0\right\}, N^{0}=l^{\infty}(\mathbf{N}, M) / I_{0}$. It is easy to see that $M^{\prime} \cap N^{\omega}$ is commutative iff $M^{\prime} \cap N^{0}$ is commutative. Then one shows that if $M^{\prime} \cap N^{\omega}$ is noncommutative, there are no abelian projections in $M^{\prime} \cap N^{\omega}$, i.e. $M^{\prime} \cap N^{\omega}$ is continuous and contains therefore a $\mathrm{I}_{2}$-subfactor. This proves $1^{\circ}$, since whenever we have matrix units $\left\{e_{i j}\right\}_{1 \leq i, j \leq 2}$ in $M^{\prime} \cap N^{\omega}$, they lift to matrix units $\left\{e_{i j}^{k}\right\}_{1 \leq i, j \leq 2}$ in $N$ with $\left\{e_{i j}^{k}\right\}_{k=1}^{\infty} \in e_{i j}$. In particular we have that $\lim _{k \rightarrow \omega}\left\|\left[e_{i j}^{k}, x\right]\right\|_{2}=0,(i, j)$ fixed, $x \in M$, which implies $1^{\circ}$. 
To prove the result about the nonexistence of abelian projections in a noncommutative algebra $M^{\prime} \cap N^{\omega}$, we take a dense sequence $\left(x_{k}\right)_{k=1}^{\infty}$ in $(M)_{1}$ and put

$$
N_{k}=\left\{x \in(N)_{1} \mid\left\|\left[x, x_{i}\right]\right\|_{2}<1 / k, 1 \leq i \leq k\right\} .
$$

As in [McD, Lemma 6], we get the following result:

Suppose $M^{\prime} \cap N^{0}$ is noncommutative. Let $f \in N_{3 k}$ be a projection of trace $\lambda \neq 0$. Then there is an $\alpha(\lambda)>0$ and $y, z \in N_{k}$ such that $y=f y f, z=f z f$ and $\|[y, z]\|_{2}>\alpha(\lambda)$. Moreover, $\alpha(\lambda)$ is independent of $\mathrm{k}$.

This completes the proof of the theorem. Q.E.D.

\section{REFERENCES}

[Ak] C. Akemann, The dual space of an operator algebra, Trans. Amer. Math. Soc. 126 (1967), 286-302.

[Be] E. Bédos, On actions of amenable groups on $\mathrm{II}_{1}$-factors, Preprint 1988.

[Co1] A. Connes, Classification of injective factors, Cases $\mathrm{II}_{1}, \mathrm{II}_{\infty}, \mathrm{III}_{\lambda}, \lambda \neq 1$, Ann. of Math. 104 (1976), 73-115.

[Co2] _ Outer conjugacy classes of automorphisms of factors, Ann. Sci. École Norm. Sup. 8 (1975), 383-420.

[CoFW] A. Connes, J. Feldman and B. Weiss, An amenable equivalence relation is generated by a single transformation, Ergodic Theory Dynamical Systems 1 (1981), 431-450.

[Jo] V. F. R. Jones, Index of subfactors, Invent. Math. 72 (1983), 1-25.

[Oc] A. Ocneanu, Quantized group string algebras and Galois theory for algebras, preprint.

[Po1] S. Popa, A short proof of "Injectivity implies Hyperfiniteness" for finite von NeumannAlgebras, J. Operations Theory 16 (1986), 261-272.

[Po2] _ Maximal injective subalgebras in factors associated with free groups, Adv. in Math. 50 (1983), 27-48.

[Po3] __ Classification of subfactors: reduction to commuting squares, Invent. Math. (to appear).

[McD] D. McDuff, Central sequences and the hyperfinite factor, Proc. London Math. Soc. (3) 21 (1970), 443-461.

[MvN] F. Murray and J. von Neumann, Rings of operators IV, Ann. of Math. 44 (1943), 716-808.

[Ta1] M. Takesaki, On the singularity of a positive linear functional, Proc. Japan Acad. 35 (1959), 365-366.

[Ta2] _ _ Theory of operator algebras I, Springer, Berlin, Heidelberg, and New York, 1979.

Department of Mathematics, University of California at los Angeles, 405 Hilgard Avenue, Los ANgeles, California 90024-1555 